\title{
The use of neuralgia medications to treat sensory neuropathic cough: our experience in a retrospective cohort of thirty-two patients
}

Zachary J. Bastian, Robert W. Bastian

Objective: This study sought to: 1) quantify response rate and efficacy of amitriptyline, desipramine, and gabapentin in treating sensory neuropathic cough; and 2) describe an efficient treatment protocol. Study Design: This study is a retrospective case series.

Methods: Persons diagnosed with sensory neuropathic cough during a one-year period were potential study candidates. To bolster the diagnosis credibility, only persons who had been treated elsewhere for gastroesophageal reflux disease, asthma, and allergy with no reduction of cough were included. Upon diagnosis of sensory neuropathic cough, each person was treated with either amitriptyline, desipramine, or gabapentin, titrating the dose upward to desired benefit or the dose limit. If the benefit was insufficient, another of the medications was used next, using a similar dose escalation strategy. Data points included patient demographics, initial and final medication, final dose, and degree of improvement. Results: 32 patients met the diagnostic and inclusion criteria and had a complete data set. $94 \%$ (30 of 32) of the patients responded to at least one of the medications. The 32 patients undertook a total of 45 single-medication trials. Patients reported symptom relief during $78 \%$ (14 of 18 ) of amitriptyline trials, $73 \%$ (11 of 15 ) of desipramine trials, and $83 \%$ (10 of 12) of gabapentin trials. At final dosage, symptom reduction averaged $77 \%$ on amitriptyline, $73 \%$ on desipramine, and $69 \%$ on gabapentin. Conclusion: Amitriptyline, desipramine, and gabapentin appear to vary in their effectiveness for individual cases of sensory neuropathic cough; across a whole cohort, symptom relief was similar in frequency and degree on any of the three medications. More evidence is needed to demonstrate more convincingly the effectiveness of these medications, but this data set suggests that each of these three medications deserves consideration in the codified treatment protocol presented here. 


\section{Authors:}

3 Zachary J. Bastian, MB ChB

Department of Otolaryngology-Head and Neck Surgery, University of North Carolina, Chapel

6 Robert W. Bastian, MD

7 Bastian Voice Institute, Downers Grove, Illinois, United States of America

8 Clinical Professor, Department of Otolaryngology, Head and Neck Surgery, Loyola University Medical Center, Maywood, Illinois, United States of America

10 Institution: Bastian Voice Institute, Downers Grove, Illinois, United States of America

12 The corresponding author is Robert W. Bastian, MD.

Address: Bastian Voice Institute, 3010 Highland Parkway, Suite 550, Downers Grove, IL 60515.

Phone: (630) 724-1100. Fax: (630) 724-0084.

Email: robert.bastian@bastianvoice.com 


\section{INTRODUCTION}

Coughing is responsible for 29.5 million visits to U.S. doctors every year (Irwin, 2006), commanding significant healthcare resources. Chronic cough can be due to diseases such as asthma, COPD, eosinophilic bronchitis, bronchiectasis, post-nasal drip, and gastroesophageal reflux disease. It also is a defining feature of sensory neuropathic cough (SNC).

SNC has been previously described, although called different names. Lee and Woo (2005) wrote about "sensory neuropathy presenting as chronic cough," and they linked this type of chronic cough to an underlying sensory disturbance by demonstrating co-incident motor neuropathy using videostroboscopy and electromyography. Bastian, Vaidya, and Delsupehe (2006) described SNC in detail as a clinical syndrome and proposed a set of diagnostic criteria. Vertigan and Gibson (2010) studied sensory disturbances and triggers (a feature of the SNC diagnosis) in a group of patients with refractory chronic cough. A review article by Gibson and Ryan (2010) includes sensory neuropathy in the diagnostic framework for chronic cough.

SNC sometimes develops following an upper respiratory infection, a herpes zoster outbreak in the pharynx, or thyroid or cervical spine surgery. Such cases provide insight into its mechanism. It behaves like vagal or glossopharyngeal neuralgia, but instead of pain, the nerve damage or dysfunction causes an intermittent, abrupt-onset sensation that initiates the coughing. In general, the sensation itself is virtually the same each time, though its perceived urgency may vary. Also common are patientspecific triggers such as talking, a loud laugh, attempting to sing a high note, taking a deep breath, breathing in cold air, touching a specific spot on the skin of the neck, and change of body position. While some cases have the apparent etiologies described above, a large number of patients develop SNC without an identifiable antecedent explanation. 
This article reviews thirty-two sequential SNC patients in order to: 1) Compare the response rates and efficacy of three neuralgia medications (amitriptyline, desipramine, gabapentin) for treating SNC, and 2) Describe an efficient treatment protocol for SNC.

\section{MATERIALS \& METHODS}

Ethics approval (protocol \#5460) for this case series was obtained from the institutional review board of Advocate Healthcare. Consent was waived for this retrospective study. All new patients diagnosed with SNC during the 12-month period spanning March 1, 2011 to February 28, 2012 were screened for inclusion. The SNC diagnoses had all been based on previously published criteria (Bastian, Vaidya \& Delsupehe, 2006), including: cough duration of at least eight weeks; recurrent sensory disturbances of instantaneous onset (e.g., a tickle) immediately before each cough episode; neuralgia-like triggers (e.g., talking, cold air or warm air); dozens to hundreds of daily cough episodes; and non-productive cough. In none of these cases was there an attempt to obtain direct evidence of damage to sensory nerves, whether via clinical electrophysiology, biopsy, autopsy, or other means. No systemic evaluation of tic disorders or chronic somatization was performed, but patients we diagnosed with coughing tics or psychogenic cough were excluded from this series.

These SNC patients were screened for inclusion in this study based on the following additional and more stringent criteria:

- No other laryngologic diagnosis;

- Cough duration of at least five months;

- Prior treatment elsewhere for gastroesophageal reflux disease, asthma, and allergy as potential explanations for the chronic cough, using (respectively) proton pump inhibitors, asthma inhalers, and antihistamines, all with zero reported benefit.

These criteria produced 32 includable patients. 
Table 1 details the treatment protocol for SNC developed and used by the senior author for over

6615 years. Table 2 summarizes the various sequences of medication trials conducted among the group of $67 \quad 32$ patients.

Percent reduction of symptoms reported by the patient was this paper's measure for degree of efficacy. Although not validated, it was published previously (Bastian, Vaidya \& Delsupehe, 2006) to discuss the response of SNC to amitriptyline, and has been used by the senior author over at least 15

71 years of treating this entity.

During the course of a treatment trial, patients (often with third-party input) were asked periodically to report the percentage by which their original coughing symptoms had been reduced, considering together the original frequency, duration, and severity of their coughing episodes. The final assessment point was a minimum of six months after the start of the first neuralgia medication trial.

\section{RESULTS}

The cohort's median age was 63, with an age range of 23 to 80 years. Female patients outnumbered male patients $3: 1$. The median duration of cough was 60 months, with a range of five to 432 months. The most common abrupt sensory disturbance preceding cough was a tickle experienced at the level of the sternal notch. An antecedent respiratory tract infection, remembered as specifically tied temporally to the onset of the cough, was reported by $38 \%$ of patients, with coughing continuing after the infectious symptoms resolved. Nighttime episodes awakening from sleep were reported by $28 \%$ of patients, and $16 \%$ reported at least occasional laryngospasm in association with severe episodes. Many patients also noted a crescendo of symptoms for several months after each subsequent upper respiratory infection.

Table 3 details patients' final reported degree of response after one or more medication trials.

The "no response" category includes one patient who had no response after trying amitriptyline and desipramine but who never tried gabapentin. 
Table 4 details the cohort's response to individual medications. Responders were those who had

90 a percent reduction of symptoms greater than 0 . The median dose data shows that most patients needed

91 to titrate upwards from initial dosing for maximal benefit. The data show response rates for

92 amitriptyline and desipramine (usually given first) of $78 \%$ and $73 \%$, respectively, and of $83 \%$ for

93 gabapentin. They also show degrees of efficacy (by percent reduction of symptoms) ranging from $77 \%$

94 for amitriptyline to $73 \%$ for desipramine to $69 \%$ for gabapentin.

\section{DISCUSSION}

96

Sensory neuropathic cough is diagnosed clinically, using the criteria mentioned above, and as the literature shows, it is effectively treated with neuralgia medications. Lee and Woo (2005) found gabapentin to be effective in the treatment of neuropathic chronic cough. Jeyakumar, Brickman, and Haben (2006) did a randomized control trial using codeine plus guaifenesin versus the neuralgia medication amitriptyline for patients with a non-productive, intractable cough, and found amitriptyline to be superior. Halum, Sycamore, and McRae (2009) reported a retrospective series in which pregabalin was effective for patients with laryngeal sensory neuropathy. A randomized, double blind, placebo-controlled trial done by Ryan, Birring, and Gibson (2012) demonstrated gabapentin's effectiveness for chronic refractory cough. Another paper described a cohort of patients with chronic idiopathic cough treated to good effect with gabapentin (Van der Kerhove et al, 2012). Norris and Schweinfurth (2010) treated chronic cough patients with amitriptyline, using gabapentin second line, and found this regimen worked well.

Most patients in our cohort and in prior reports took amitriptyline at doses of only $10-40 \mathrm{mg}$ daily (Jeyakumar et al, 2006; Norris and Schweinfurth, 2010). Low-dose tricyclics are not uncommon in treating neuropathic pain (Moulin et al, 2014; Table 1), but most patients in controlled studies took much higher doses, e.g., 100-150mg/d (Hearn et al, 2014; Derry et al, 2015). Similarly, duloxetine and venlafaxine are effective for neuropathic pain primarily at higher doses (Lunn, Hughes, and 
113 Wiffen, 2014; Rowbotham et al, 2004). Since desipramine and amitriptyline have multimodal effects

114 on the nervous system, their mechanism of action in SNC is not clear.

115 Left untreated, sensory neuropathic cough can profoundly affect a patient's quality of life.

116 While the dozens to hundreds of brief coughing episodes each day can annoy and frustrate, most

117 patients also experience at least a few more severe and potentially humiliating episodes each day,

118 lasting from 10 seconds to several minutes, and associated with rhinorrhea, oculorrhea, retching,

119 vomiting, laryngospasm, near-syncope, and urinary incontinence. In the senior author's practice, some

120 patients have even reported cough-induced rib fracture, subconjunctival hemorrhage, and progressive

121 pelvic floor weakening.

122

This disorder requires patience to treat and can be very labor-intensive. Many phone calls may

123 be traded between patient and physician, with office staff serving as intermediaries, but typically a

124 single visit and four or five episodes of subsequent phone management suffice. Patients who have

125 become jaded or even contemptuous of clinician help after years of searching must be urged to

126 persevere through one or more medication trials.

In this cohort, no medication emerged as clearly superior in the treatment of SNC, though we

128 did not statistically verify that these medications' efficacies were truly similar to one another, nor did

129 we statistically analyze the sample sizes to determine what level of confidence could be placed in the

130 effect of these medications. Interestingly, a paper comparing the efficacy of neuralgia medications for

131 diabetic neuropathy among patients randomized and placebo-controlled to receive amitriptyline,

132 duloxetine, or pregabalin also found that no one medication emerged as superior (Boyle et al, 2012).

133 This paper appears to be the first to present a clinical protocol for treating SNC. An effective

134 treatment protocol must efficiently move the patient through a series of different medications,

135 individualizing according to patient particulars, until a sufficient response is achieved. To accomplish

136 this based upon a single patient visit, the physician carefully educates the patient and family up front 
137 through personal discussion, teaching videos, and written handouts, so that he or she can follow up

138 effectively by phone. Regarding our gabapentin trial protocol, note that a QID interval works better in

139 our experience than gabapentin's usual TID interval, as many patients using a TID interval experience

140 breakthrough coughing just before the next dose.

141 A prospective, randomized study with placebo controls would go further than our study to

142 verify the relative efficacy of these three neuralgia medications. Still, as mentioned already, it should

143 be noted that this cohort's median cough duration was 60 months, during which numerous other

144 medications (including treatments for gastroesophageal reflux disease, asthma, and allergy) had been

145 tried for each patient reported here, without any perceived benefit. Also, the final assessment point was

146 a minimum of six months after onset of treatment with medication for sensory neuropathy, and often

147 much longer. Furthermore, others have employed randomization and a control group and found

148 amitriptyline and gabapentin, respectively, to be effective for treating SNC (Jeyakumar, Brickman \&

149 Haben, 2006; Ryan, Birring \& Gibson, 2012).

150 Efficacy data was based on patient report of overall percent reduction of symptoms, which was

151 the only efficacy-related data available in this retrospective report. In the senior author's experience,

152 this form of patient response is easily understood by most patients and is clinically efficient and

153 powerful, but it is a non-validated measure and less precise than some other cough measures, and thus

154 is another limitation of this study.

\section{CONCLUSION}

156 SNC is emerging as a distinct syndromic, diagnostic category for chronic cough. For this

157 diagnosis, first-line agents amitriptyline and desipramine have response rates and degrees of efficacy

158 that appear to be similar. Gabapentin also offers a comparable response rate, though with less

159 convenient dosing. Thorough up-front education combined with phone follow-up is a good way to 
160 work with this labor-intensive group of patients, who often need more than one treatment trial with 161 different neuralgia medications to achieve optimal benefit. 


\section{ACKNOWLEDGMENTS}

163 Tim Avery, a technical and writing assistant with the senior author's medical practice, provided

164 copyediting and content suggestions.

165

166 
167

168

169

170

171

172

173

174

175

176

177

178

179

180

181

182

183

184

185

186

187

188

189

\section{REFERENCES}

All references were found on PubMed.

Bastian RW, Vaidya AM, Delsupehe KG. 2006. Sensory neuropathic cough: a common and treatable cause of chronic cough. Otolaryngol Head and Neck Surg 135(1): 17-21.

Boyle J, Eriksson ME, Gribble L, Gouni R, Johnsen S, Coppini DV, Kerr D. 2012. Randomized, placebo-controlled comparison of amitriptyline, duloxetine, and pregabalin in patients with chronic diabetic peripheral neuropathic pain: impact on pain, polysomnographic sleep, daytime functioning, and quality of life. Diabetes Care 35(12): 2451-2458.

Derry S, Wiffen PJ, Aldington D, Moore RA. 2015. Nortriptyline for neuropathic pain in adults. Cochrane Database Syst Rev 2015(1): CD011209.

Gibson PG, Ryan NM. 2011. Cough pharmacotherapy: Current and future status. Expert Opin Pharmacother 12(11): 1745-1755.

Halum SL, Sycamore DL, McRae BR. 2009. A new treatment option for laryngeal sensory neuropathy. Laryngoscope 119:1844-1847.

Hearn L, Moore RA, Derry S, Wiffen PJ, Phillips T. 2014. Desipramine for neuropathic pain in adults. Cochrane Database Syst Rev 2014(9): CD011003.

Irwin RS. 2006. Introduction to the diagnosis and management of cough: ACCP evidence-based clinical practice guidelines. Chest 129(1 Suppl): 25S-27S.

Jeyakumar A, Brickman TM, Haben M. 2006. Effectiveness of amitriptyline versus cough suppressants in the treatment of chronic cough resulting from postviral vagal neuropathy. Laryngoscope 116: 2108-2112.

Lee B, Woo P. 2005. Chronic cough as a sign of laryngeal sensory neuropathy: diagnosis and treatment. Ann Otol Rhinol Laryngol 114: 253-257. 
190 Lunn MP, Hughes RA, Wiffen PJ. 2014. Duloxetine for treating painful neuropathy, chronic pain or 191 fibromyalgia. Cochrane Database Syst Rev 2014(1): CD007115.

192 Moulin D, Boulanger A, Clark AJ, Clarke H, Dao T, Finley GA, Furlan A, Gilron I, Gordon A, 193 Morley-Forster PK, Sessle BJ, Squire P, Stinson J, Taenzer P, Velly A, Ware MA, Weinberg

194 EL, Williamson OD. 2014. Pharmacological management of chronic neuropathic pain: revised 195 consensus statement from the Canadian Pain Society. Pain Res Manag 19(6): 328-35.

196 Norris BK, Schweinfurth JM. 2010. Management of recurrent laryngeal sensory neuropathic 197 symptoms. Ann Otol Rhinol Laryngol 119(3): 188-191.

198 Rowbotham MC, Goli V, Kunz NR, Lei D. 2004. Venlafaxine extended release in the treatment of 199 painful diabetic neuropathy: a double-blind, placebo-controlled study. Pain 110(3): 697-706. 200 Ryan NM, Birring SS, Gibson PG. 2012. Gabapentin for refractory chronic cough: a randomized, 201 double-blind, placebo-controlled trial. Lancet 380(9853): 1583-9.

202 Van de Kerkhove C, Goeminne PC, Van Bleyenbergh P, Dupont LJ. 2012. A cohort description and 203 analysis of the effect of gabapentin on idiopathic cough. Cough 8(9).

204 Vertigan AE, Gibson PG. 2010. Chronic refractory cough as a sensory neuropathy: Evidence from a 205 reinterpretation of cough triggers. $J$ Voice 25(5): 596-601. 


\section{Table $\mathbf{1}$ (on next page)}

Treatment protocol for sensory neuropathic cough

*Capsaicin spray is sometimes used for those who fail all medication trials. It is a topical spray administered to the posterior oropharynx. It appears to work either by gradually depleting substance $p$ and thereby lowering the threshold for cough triggers, or as a counterirritant to abort attacks in progress. 
Step 1: First-line medication trial

- Medication: amitriptyline or desipramine; amitriptyline for patients 60 years old or younger and, due to potentially lesser side effects, desipramine for patients older than 60 (as a general guideline)

- Trial method: Take $10 \mathrm{mg}$ daily, two hours before bed, for the first day or two. Then, until symptoms are decreased $80 \%$ or more, or until a maximum dose of $80 \mathrm{mg}$ is reached, continue increasing the daily dose by $10 \mathrm{mg}$ every one or two days. If breakthrough cough is clearly evident in afternoon and evening, add 10 or $20 \mathrm{mg}$ at noon.

Step 2: Phone follow-up, either 14 days after starting the medication, or sooner, if the patient has forgotten the instructions or has a concern

- At every phone follow-up, the patient must supply: name of current medication, dose, and duration of use; percent reduction of symptoms globally; side effects, if any; questions, if any; and best contact information. Staff records this information for physician review and response.

- Physician tells staff to instruct the patient to continue increasing dose (if the patient notices any reduction in cough symptoms whatsoever), possibly with instructions for adjusting the logistics of use (for instance, adding a booster of dose of 10 or $20 \mathrm{mg}$ if the patient has breakthrough coughing at a certain time of day), or else to taper off current medication and then to begin another medication (if the current medication's dose limit has been reached, or if side effects have become unacceptable, without providing satisfactory benefit to the patient).

Step 3: Second-line medication trial (if needed)

- Medication: gabapentin (best to take with food, except at bedtime) 
- Trial method:

- Days 1-3: $300 \mathrm{mg}$ at bedtime

- Days 4-6: $300 \mathrm{mg}$ at lunch and bedtime (600 mg total)

- Days 7-9: $300 \mathrm{mg}$ at breakfast, mid-afternoon, bedtime (900 mg total)

- Days 10-12: $300 \mathrm{mg}$ at breakfast, lunch, dinner, bedtime (1200 mg total)

- After Day 12: If insufficient response, patient may (as side effects permit) add another $300 \mathrm{mg}$ to one of the daily 300-mg doses every three days, up to a maximum daily dose of $2400 \mathrm{mg}$ (four 600-mg doses)

- $\quad$ Repeat Step 2.

Step 4: Third-line, fourth-line, fifth-line, etc. medication trials (if needed)

- Citalopram, pregabalin, oxcarbazepine

- Capsaicin spray*

Step 5: Gradual discontinuation trial (optional)

- An option for patients who achieve $80 \%$ or greater reduction of symptoms for at least two months

- Some patients will need long-term treatment, and some patients remit and relapse, with relapses often following upper respiratory infections 


\section{Table 2 (on next page)}

Courses of the treatment trials

$\mathrm{n}=32$ patients; final medication tried in bold*As mentioned in the footnote to Table 3 , one of these patients went on to use capsaicin spray, with benefit. 


\begin{tabular}{|l|l|l|l|}
\hline $\begin{array}{l}\text { Number of } \\
\text { Patients }\end{array}$ & First Medication & $\begin{array}{l}\text { Second } \\
\text { Medication }\end{array}$ & Third Medication \\
\hline 12 & amitriptyline & & \\
\hline 7 & desipramine & & \\
\hline 6 & desipramine & gabapentin & \\
\hline $3^{*}$ & amitriptyline & gabapentin & \\
\hline 1 & amitriptyline & desipramine & gabapentin \\
\hline 1 & amitriptyline & desipramine & \\
\hline 1 & amitriptyline & gabapentin & $\begin{array}{l}\text { amitriptyline }+ \\
\text { gabapentin }\end{array}$ \\
\hline 1 & gabapentin & & \\
\hline
\end{tabular}




\section{Table 3(on next page)}

Response quartiles, for patients at final medication and dose

$n=32$ patients* One of these two patients went on to achieve $80 \%$ reduction of cough symptoms using capsaicin spray; note the footnote to Table 1 regarding capsaicin spray. The other patient tried amitriptyline and desipramine without benefit and never went on to a gabapentin trial. 


\begin{tabular}{|l|l|l|l|l|l|l|}
\hline & $\begin{array}{l}\text { No } \\
\text { response }\end{array}$ & $\begin{array}{l}\text { Subtle } \\
1-25 \% \\
\text { Reduction } \\
\text { of } \\
\text { Symptoms }\end{array}$ & $\begin{array}{l}\text { Small } \\
26-50 \% \\
\text { Reduction } \\
\text { of } \\
\text { Symptoms }\end{array}$ & $\begin{array}{l}\text { Medium } \\
51-75 \% \\
\text { Reduction } \\
\text { of } \\
\text { Symptoms }\end{array}$ & $\begin{array}{l}\text { Large } \\
76-100 \% \\
\text { Reduction } \\
\text { of } \\
\text { Symptoms }\end{array}$ & $\begin{array}{c}\text { Total } \\
\text { number }\end{array}$ \\
\hline $\begin{array}{l}\text { Number of } \\
\text { patients }\end{array}$ & $2 *$ & 2 & 7 & 5 & 16 & $\mathbf{3 2}$ \\
\hline Percentage & 6 & 6 & 22 & 16 & 50 & $\mathbf{1 0 0}$ \\
\hline
\end{tabular}




\section{Table 4(on next page)}

Medication response

$\mathrm{n}=45$ treatment trials, taken by the 32 patients (some patients tried more than one medication)Excluded from this table is a 46th trial in which one of the patients, after trying amitriptyline and gabapentin separately, took amitriptyline and gabapentin together, and achieved a $25 \%$ reduction of symptoms. 


\begin{tabular}{|l|l|l|l|l|}
\hline Medication & $\begin{array}{l}\text { Number of } \\
\text { Patients Who } \\
\text { Tried Medication }\end{array}$ & $\begin{array}{l}\text { Number / \% } \\
\text { Who } \\
\text { Responded }\end{array}$ & $\begin{array}{l}\text { Avg. \% Reduction } \\
\text { of Symptoms at } \\
\text { Final Dose }\end{array}$ & $\begin{array}{l}\text { Median Final } \\
\text { Dose for } \\
\text { Responders (mg) }\end{array}$ \\
\hline amitriptyline & 18 & $14 / 78$ & 77 & 40 \\
\hline desipramine & 15 & $11 / 73$ & 73 & 25 \\
\hline gabapentin & 12 & $10 / 83$ & 69 & 1350 \\
\hline
\end{tabular}

